\title{
Active Testing for Face Detection and Localization
}

\author{
Raphael Sznitman \\ Department of Computer Science \\ Johns Hopkins University \\ Bruno Jedynak \\ Department of Applied Mathematics and Statistics \\ Johns Hopkins University
}

\begin{abstract}
We provide a novel search technique, which uses a hierarchical model and a mutual information gain heuristic to efficiently prune the search space when localizing faces in images. We show exponential gains in computation over traditional sliding window approaches, while keeping similar performance levels.
\end{abstract}

\section{Introduction}

In recent years, face detection algorithms have provided extremely accurate methods to localize faces in images. Typically, these have involved the use of a strong classifier, which estimates the presence of a face given a particular subwindow of the image. Successful classifiers have used Boosted Cascades [25, 22, 17, 27], Neural Networks [21, 19, 10] and SVM's [20, 24] among others.

In order to localize faces, the aforementioned algorithms have relied on a sliding window approach. The idea is to inspect the entire image by sequentially observing each and every location a face may be in by using a classifier. In most face detection algorithms [25, 17, 19, 27, this involves inspecting all pixels of the image for faces, at all possible face sizes. This exhaustive search, however, is computationally expensive and in general not scalable to large images. For example, for real-time face detection using modern cameras $(4000 \times 3000$ pixels per image), more than 100 million evaluations are required, making it hopeless on any standard computer.

To overcome this problem, previous works in object and face localization have simply reduced the pose space by allowing only a coarse grid of possible locations [3, 21, 25]. An elegant improvement to object detection was proposed in [22] where "feature-centric" evaluation are performed, as opposed to "window-centric", allowing previous computation to be reused. Such a method 
however relies on strong knowledge of the classifier used. More recently, a globally optimal branch-and-bound subwindow search method for objects in images was proposed [15] and extended to videos [28. Here, the classifier and the feature space used to locate the object are dependent on a single robust feature (e.g. SIFT [18]), making it difficult to use in the context of faces.

In this paper, we propose a novel search strategy, which can be combined with any face classifier, in order to significantly reduce the computational cost involved with searching the entire space. The design principle is as follows: We assume that a perfect face classifier is available, i.e. one which always provides the correct answer. In practice however, such a classifier does not exist and an accurate one (as in 25, 17, 19, 27]) will be used instead. Our goal is then to reduce the total number of classifier evaluations required to detect and locate faces in images, while still providing similar performance levels when compared with an exhaustive search.

A proposed strategy for computational shape recognition [8], argues that the task of visually recognizing an object can be accomplished by querying the image in a sequential and adaptive way. In general, this can be regarded as a coarse-to-fine approach to perception [1, 25, 6, 7]. This "twenty questions" approach can be described as follows: there is a fact to be verified, e.g. "is there a face in the field of view", and each query, which consists of evaluating a particular function of the image, is chosen to maximally reduce the expected uncertainty about this fact. In the context of computer vision, such approaches have led to two different types of search algorithms: offline and online. In the offline versions, the "where to look next" strategy is computed once and for all, anticipating all possible queries. It has led to efficient algorithms for symbol recognition [1, face [6] and cat 7] detection. In the online version, the strategy is computed sequentially, as information is gathered. It has led to a road tracking algorithm [8, 9]: this approach is known as Active Testing.

In this paper, we extend the active testing framework in order to do fast face detection and localization. We provide a way to ask questions that are general and specific with regard to the face pose, and span different feature spaces. Similarly to the "twenty questions" game, questions such as "is the object at this location with this size?" are asked by means of an accurate face classifier [25, 24, [19, 27, independently of what features are used to guide the search. We show here that this approach provides a coherent framework, with few parameters to choose or tune, which significantly reduces the number of classifier evaluations necessary to localize faces. Comparison of our method with state-of-the-art face detection algorithms, and the traditional sliding window approach, indicate that our framework reduces, by several orders of magnitude, the number of classifier evaluation needed while maintaining similar accuracy levels on localization and detection tasks. Even though this paper specifically focuses on frontal faces, this approach can be extended to faces in general [14, 13, 4, 16, 2], other object categories [5] and to most classifiers in the machine learning literature.

The remainder of this paper is organized as follows: in section 2, the general framework of our method is presented along with implementation details. Section 3 describes localization experiments, and in section 4 we compare the 


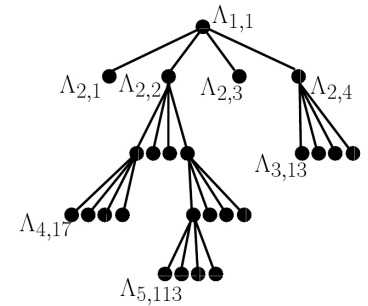

(a)

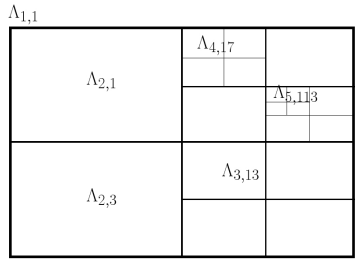

(b)

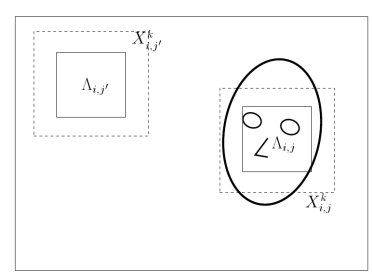

(c)

Figure 1: Each node in the tree (a) corresponds to subwindow in the image (b). The root of the tree, $\Lambda_{1,1}$, represents the entire image space and has four children $\left(\Lambda_{2,1}, \Lambda_{2,2}, \Lambda_{2,3}, \Lambda_{2,4}\right)$. (c) Example Query: Here, the face center is, $Y=l \in \Lambda_{i, j}$. The query $X_{i, j}^{k}$, counts the proportion of edges in a window twice the size of $\Lambda_{i, j}$, centered on $\Lambda_{i, j} . k$ indicates that we count the proportion of edges on a surface twice the size of the subwindow $\Lambda_{i, j}$, while $\{i, j\}$ provides the pose subset in $\Lambda$.

performance with state-of-the-art methods on a detection and localization task. Concluding remarks are provided in section 5 .

\section{Active Testing}

The goal set forth is to detect and localize a single frontal face of unknown size, which may or may not be present in the image. We define the pose of a face, as the pixel location of the face center and a face scale. That is, we treat localization as placing a bounding box around a face. In section 4 , we detail how this can be extended to searching for multiple faces.

Active Testing (AT) can be regarded as a search algorithm which uses an information gain heuristic in order to find regions of the search space which appear promising. The region which is to be observed next is determined as information is gathered, and thus can be viewed as an online variation of the "twenty questions" game. The general approach is as follows: we are looking for a face in an image, and are provided with a set of questions which help us determine where the face is located. Questions are answered with some uncertainty, reducing the search space and eventually leading to the face pose.

In addition, it is also assumed that a special question regarding the exact face pose is available. This question is treated as an "Oracle", always providing a perfect answer when queried but is computationally expensive relative to other questions. Querying the oracle at every location would provide the face pose but is expensive and inefficient as certain questions are more informative than

others and help reduce the search space faster. Consequently, a sub-goal is to determine face pose with as few questions as possible. 


\subsection{Model and Algorithm}

Let $Y=(L, S)$ be a discrete random variable defining the face pose; where $L$ is the location of the face center (i.e. pixel coordinates), and $S$ is the face scale, such that $S$ can take values $\{1, \ldots, M\}$ corresponding to $M$ face size intervals. Additionally, $Y$ can take one extra value when the face is not in the image. Let

$$
\Lambda=\left\{\Lambda_{i, j}, i=1, \ldots, D, j=1, \ldots, 4^{i-1}\right\}
$$

be a quadtree of finite size, which decomposes the image space; $i$ indexes the level in the tree and $j$ designates the cell at that level (see figure 1(a)). Every leaf is associated with a pixel in the image and each non-terminal node corresponds to a unique subwindow in the image, representing a subset of poses (figure $1(\mathrm{~b})$ ). When no face is present in the image then $Y \in \bar{\Lambda}_{1,1}$, where $\bar{\Lambda}_{1,1}$ denotes the complement of $\Lambda_{1,1}$.

We are interested in refining the estimate of where the face is located iteratively and hence denote $\pi_{t}$ as the probability density of $Y$ at iteration step t. Let $u_{i, j, s}=P\left(L \in \Lambda_{i, j}, S=s\right), \Lambda_{i, j} \subset \Lambda, s \in\{1, \ldots, M\}$. By construction, calculating $u_{i, j, s}$ can be achieved by summing the probability of $\Lambda_{i, j}$ 's children. Clearly, $u_{1,1, s}=u_{2,1, s}+u_{2,2, s}+u_{2,3, s}+u_{2,4, s}$ and similarly for any other $u_{i, j, s}$. For any node, we also denote $u_{i, j}=\pi\left(\Lambda_{i, j}\right)=\sum_{s=1}^{M} u_{i, j, s}$. Let $\mathcal{X}=\left\{\mathbf{X}^{1}, \ldots, \mathbf{X}^{K}\right\}$ be a set of question families, such that for each family $k$, $\mathbf{X}^{\mathbf{k}}=\left\{X_{i, j}^{k}, i=1, \ldots, D, j=1, \ldots, 4^{i-1}\right\}$, where $X_{i, j}^{k}$ is a query from family $k$, about the pose subset $\Lambda_{i, j}$.

The generic AT algorithm (algorithm 1) can then be seen as the following: to begin, $\pi_{0}$ and the first query are initialized (lines 1 to 2 ). Three operations are then repeated: the response is observed (line 4); the belief of the location of $Y$ is updated using the latest observation (line 5); a new query is chosen for the next iteration (line 6). The iteration is stopped when a terminating criteria is achieved (line 7). Each line is explained in detail in the following sections.

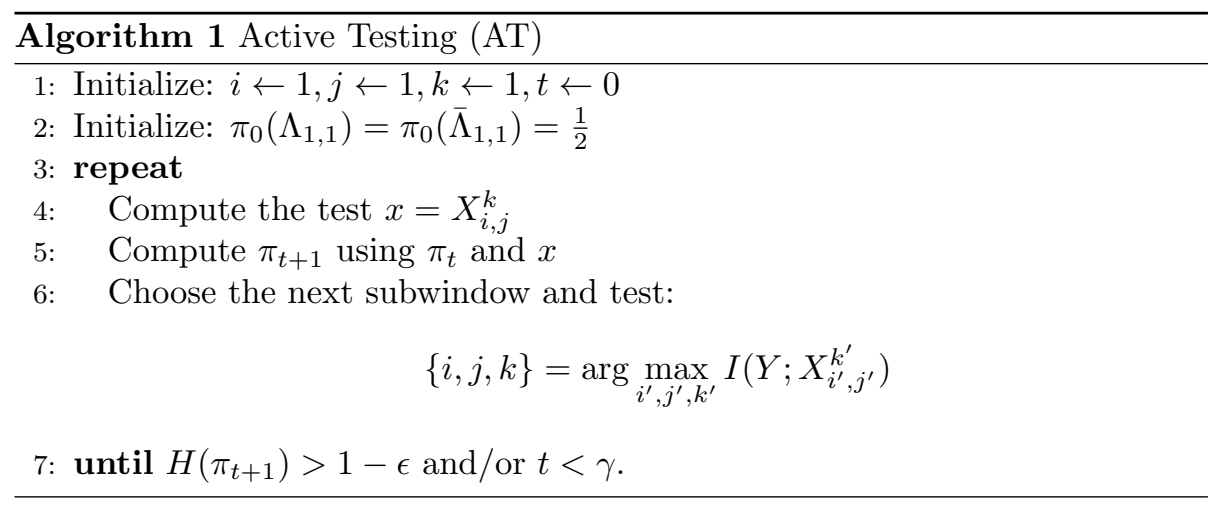




\subsection{Queries}

The AT algorithm requires a set of query families, $\mathcal{X}=\left\{\mathbf{X}^{1}, \ldots, \mathbf{X}^{K}\right\}$, to be specified. Each query family, $\mathbf{X}^{k}$, consists of evaluating a specific type of image functional indexed by $k$. Members of a family, $\mathbf{X}^{\mathbf{k}}=\left\{X_{i, j}^{k}, i=1, \ldots, D, j=\right.$ $\left.1, \ldots, 4^{i-1}\right\}$, are indexed by a pose index in $\Lambda$ (as in [7). That is, $X_{i, j}^{k}$ is an image functional, where $k$ defines a particular computation and $\{i, j\}$ specifies the pose subset. Note that these queries are generic and need not be binary. Example queries can be seen in Figure 1(c).

In addition, perfect tests - which precisely predict the presence of a face by using a classifier - are included in $\mathcal{X}$. When this test is used at a specific pose, either the classifier responds positively and the face is deemed found, or conversely, the response is negative and the face is assumed not to be at this pose. That is, we assume no uncertainty with regard to the response of this classifier.

In order to specify the joint distribution between the face pose $Y$ and queries $\mathcal{X}$, we make the following heuristic assumptions:

\section{Conditional Independence}

$$
\begin{array}{r}
P\left(\left\{X_{i, j}^{k}=x\right\}, i=1 \ldots D, j=1 \ldots 4^{i-1}, k=1 \ldots K \mid Y=(l, s)\right) \\
=\prod_{i, j, k} P\left(X_{i, j}^{k}=x \mid Y=(l, s)\right)
\end{array}
$$

\section{Homogeneity}

$$
P\left(X_{i, j}^{k}=x \mid Y=(l, s)\right)= \begin{cases}f_{s}^{k}(x ; i) & \text { if } l \in \Lambda_{i, j} \\ f_{0}^{k}(x ; i) & \text { otherwise }\end{cases}
$$

Here $f_{s}^{k}$ characterizes the "response" to the query $X_{i, j}^{k}$ when the center of the face is within $\Lambda_{i, j}$ with size $s$. Similarly, $f_{0}^{k}$ is the "response" when the center is not in $\Lambda_{i, j}$. Additionally, even though $K N$ queries are specified, where $N$ is the number of nodes in $\Lambda$, the number of densities needed is only $K D$. That is, for each test family, only one density per level of $\Lambda$ needs to be specified. This is why $f_{s}^{k}(\cdot, i)$ is only indexed by $i$.

Note that these assumptions are a simple way to make the problem tractable: for example, the conditional independence of queries given the location of the object $Y$ assumption is clearly a simplification as the same pixel values are used to compute many queries at different levels of $\Lambda$. Similarly, the actual responses to tests might in fact depend on the precise location of the face within $\Lambda_{i, j}$. The homogeneity assumption simplifies the response model by assuming a single model for all cases. Even when using these assumptions however, the experiments conducted here (sections 3 and 4 ) indicate that these simplifications provide a good way to solve the problem at hand. In addition, this model should be taken into account when choosing queries to use: similarly to a Naive Bayes model, queries should be individually informative. 


\subsection{Belief Update}

Once an observation has been made, the new distribution of the face location $Y$ must be calculated (line 5 of AT). At initialization (line 1 of $\mathrm{AT}$ ), $\pi_{0}\left(\Lambda_{1,1}\right)=\pi_{0}\left(\bar{\Lambda}_{1,1}\right)=\frac{1}{2}$, indicating that a face is believed to be in the image with probability $1 / 2$. Note that the probability $\pi_{0}\left(\Lambda_{1,1}\right)$ is uniformly distributed within $\Lambda_{1,1}$ by construction. Given $\pi_{t}$ and the query response $X_{i, j}^{k}=x$ at time step $t$, the updated distribution $\pi_{t+1}$ can then be calculated by using Bayes formula

$$
\pi_{t+1}(l, s)=\frac{P\left(X_{i, j}^{k}=x \mid Y=(l, s)\right) \pi_{t}(l, s)}{\sum_{s^{\prime}} \int_{l^{\prime}} P\left(X_{i, j}^{k}=x \mid Y=\left(l^{\prime}, s^{\prime}\right)\right) \pi_{t}\left(l^{\prime}, s^{\prime}\right) d l^{\prime}}
$$

Using assumptions 1 and 2 then

$$
P\left(X_{i, j}^{k}=x \mid Y=(l, s)\right)=f_{0}^{k}(x, i) \mathbb{I}_{\bar{\Lambda}_{i, j}}(l)+f_{s}^{k}(x, i) \mathbb{I}_{\Lambda_{i, j}}(l)
$$

Let us now define the likelihood ratio as

$$
r(x, s)=\frac{f_{s}^{k}(x, i)}{f_{0}^{k}(x, i)}, s=1 \ldots M
$$

then equation 3 can be written as,

$$
\pi_{t+1}(l, s)=\frac{1}{\mathcal{Z}(x)}\left(\mathbb{I}_{\bar{\Lambda}_{i, j}}(l)+\mathbb{I}_{\Lambda_{i, j}}(l) r(x, s)\right) \pi_{t}(l, s)
$$

where $\mathcal{Z}(x)$ is the normalizing constant,

$$
\mathcal{Z}(x)=\pi_{t}\left(\bar{\Lambda}_{i, j}\right)+\sum_{s=1}^{M} r(x, s) \pi_{t}\left(\Lambda_{i, j}\right)
$$

Note that the evolution from $\pi_{t}$ to $\pi_{t+1}$ only relies on $r(x)$ and allows for probability mass to be shifted onto or away from $\Lambda_{i, j}$, depending on the response of $X_{i, j}^{k}$.

In order to reduce the number of nodes to update, only a subtree is maintained, where only nodes which have probability greater than some threshold $\tau$ are included. By construction of $\Lambda$, parent nodes have probability equal to the sum of their children, hence any node which has probability larger than $\tau$ also has parent with probability greater than $\tau$. This guarantees that applying this threshold forms a subtree within $\Lambda$ containing $\Lambda_{1,1}$. This approximation of $\pi_{t}$ allows for a compact representation of the distribution.

\subsection{Query Selection}

We choose to select the next query by maximizing the mutual information gain between $Y$ and the possible queries $X_{i, j}^{k}$ (line 6 of AT). This can be written as

$$
I\left(Y ; X_{i, j}^{k}\right)=H\left(X_{i, j}^{k}\right)-H\left(X_{i, j}^{k} \mid Y\right)
$$


where,

$$
H\left(X_{i, j}^{k}\right)=h\left(\sum_{s=0}^{M} u_{i, j, s} f_{s}^{k}(\cdot)\right)
$$

here, $h(f)$ is the differential Shannon entropy of the density $f$. We simplify this expression by substituting $h(f)$ with the Gini Index [11]. The mutual information then becomes

$$
I\left(Y ; X_{i, j}^{k}\right)=\sum_{s=0}^{M} \sum_{m>s}^{M} u_{i, j, s} u_{i, j, m} \int\left(f_{s}^{k}-f_{m}^{k}\right)^{2}
$$

where $u_{i, j, 0}=1-u_{i, j}$. Note that the term $\int\left(f_{s}^{k}-f_{m}^{k}\right)^{2}$ is the Euclidean distance between the densities $f_{s}^{k}$ and $f_{m}^{k}$, and only needs to be computed once and then stored for fast evaluation.

Since we are interested in choosing both the region $\Lambda_{i, j} \in \Lambda$ and a query family $k$ which maximizes the information gain, one can simply evaluate $I\left(Y ; X_{i, j}^{k}\right)$ for all possible values of the triple $(i, j, k)$ and select the parameters providing the largest gain. However, as described in section 2.3. only a small subset of poses is ever considered at any iteration. For example, nodes which have little probability will surely only provide a small information gain. Consequently, we only need to evaluate equation 10 for the explicitly maintained subtree (Figure 1(a)). Additionally once a query has been chosen, it is removed from the set of possible queries, further reducing the amount of computation.

\subsection{Terminating Criteria}

At line 7 of the AT algorithm, two terminating criteria are presented: (i) the algorithm runs until the entropy of $\pi, H(\pi)$, is very high, (ii) the algorithm iterates for a fixed number of steps, $\gamma$. In the first case, running until the entropy is high corresponds to two possible outcomes: either a face has been found and most of the probability mass is at a single leaf of $\Lambda$ or most of the mass is outside the image, $\bar{\Lambda}_{1,1}$ and no face is believed to be present in the image. In general, the choice of which criteria to use ((i), (ii) or both) is for the user to decide. Sections 3 and 4 show the behavior of these scenarios.

In addition, for all cases, the total number of queries is bounded by the size of the tree and the number of query families. As the algorithm iterates and the classifier is queried, the number of poses with strictly positive probability

decreases. This provides a guarantee that, in the worst case, the face will be found after having observed all the poses.

\subsection{Implementation}

We now provide some implementation details and give a more in depth algorithm for updating $\pi$ (see algorithm 2) and choosing queries. 


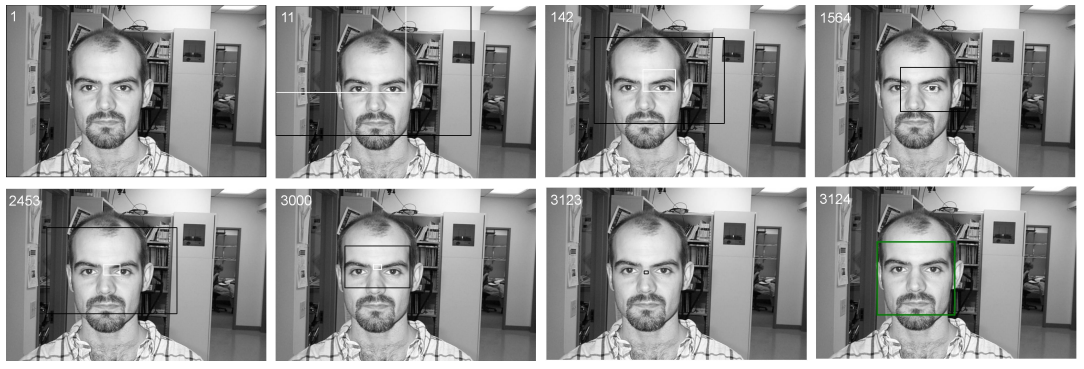

Figure 2: Sequence of queries posed by the Active Testing algorithm on a test image from the Caltech Frontal Face Dataset. In each image, a test $X_{i, j}^{k}$ is computed: white boxes show the pose, $\Lambda_{i, j}$, queried while black boxes show the subimage queried. The number indicated in the top left of each image is the iteration number of the AT algorithm. In image 3123, the Boosted Cascade is evaluated and a face is found at a given scale (green box).

Before the AT algorithm begins, all features necessary to evaluate queries from $\mathcal{X}$ for a given image are computed and stored in the form of an integral image making the evaluation of a query $O(1)$ operations (similar to [15]). This is particularly efficient since queries $X_{i, j}^{k}$ compute nested subwindows.

In order to form and maintain the subtree of $\Lambda$ (line 7 ), only nodes which are above a threshold $(\tau=0.001)$ are explicitly stored. To do this, we construct $\Lambda$ as a quadtree, and maintain a frontier set $\mathcal{F}$. $\mathcal{F}$ consists of any node $\Lambda_{i, j}$ with $u_{i, j}>\tau$ and with all children having $u_{i+1, j^{\prime}}<\tau$. Applying this rule at each iteration ensures that the maintained subtree is relevant to where the face is believed to be located. Additionally, since the probability associated at any node in the tree is equal to the sum of its children, we only need to update nodes in $\mathcal{F}$, and recurse through the tree to update the remaining nodes in $\Lambda$.

After having computed the query $X_{i, j}^{k}$, updating any node $\Lambda_{i^{\prime}, j^{\prime}} \in \mathcal{F}$ is simple: if $\Lambda_{i^{\prime}, j^{\prime}} \in \Lambda_{i, j}$, then $u_{i^{\prime}, j^{\prime}}=r(y) u_{i^{\prime}, j^{\prime}} / \mathcal{Z}$, otherwise $u_{i^{\prime}, j^{\prime}}=u_{i^{\prime}, j^{\prime}} / \mathcal{Z}$. Doing so updates $\pi$ as described in equation (6) in an efficient way. In addition, at any point in the updating of $\pi$, the next best query, $S$, seen so far is maintained. The denominator $\mathcal{Z}$ is calculated once and for all, and used to calculate equation 10 when each node is visited. Only the best score is kept, and ultimately chosen for the following iteration of the AT algorithm. That is, we compute equations (6) and (10) one after the other, requiring only one pass through the subtree per iteration.

\section{Face Localization}

To demonstrate that this framework can be used to significantly reduce the number of classifier evaluations required when searching for a face in an image, we begin by evaluating the AT algorithm on a pure localization task (as done in [15]). In the following set of experiments, each image contains exactly one 


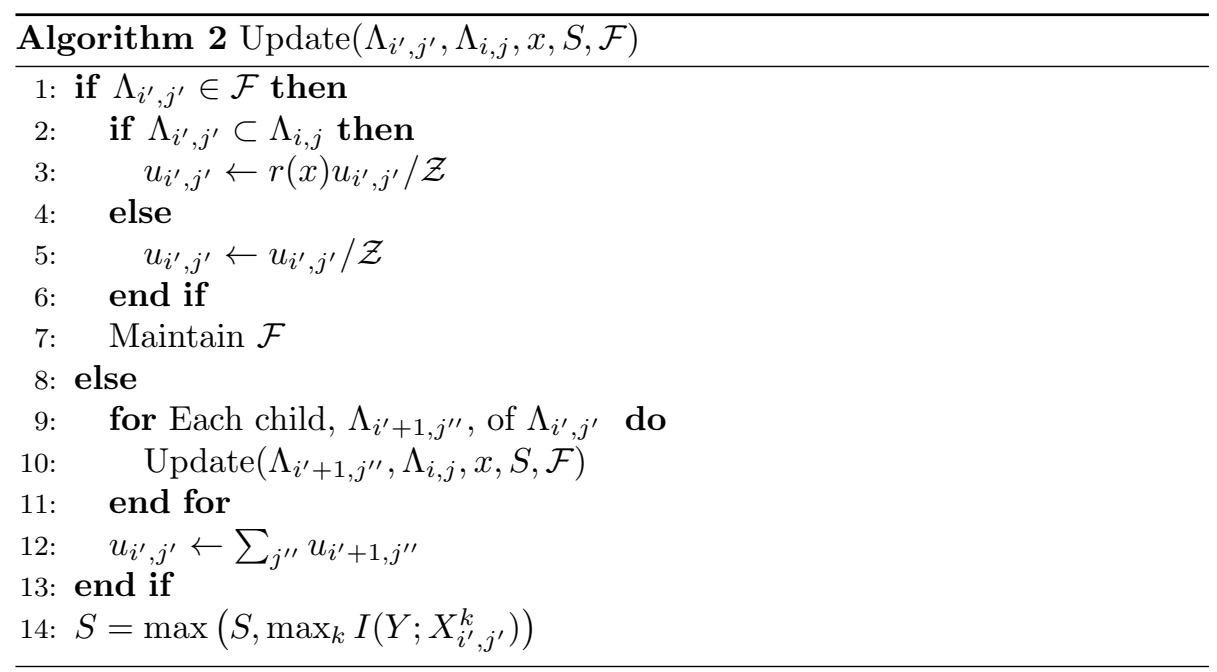

face. We describe in section 3.1 the queries used to localize faces. In section 3.3 we show how AT performs in terms of time, number of classifier evaluations and accuracy.

We perform the following experiments on the Caltech Frontal Face dataset [26], which consists of 450 images $(896 \times 592$ pixels), each containing exactly one of 27 different faces in variously cluttered environments and illuminations. Face sizes range from approximately 100 to 300 pixels in width. We choose $M=4$ possible face size intervals ([100,150], [150, 200], [200, 250], [250, 300]). All experiments are conducted on a 2.0 Gigahertz machine.

\subsection{Face Queries}

To locate faces, we first specify the following set of test families, $\mathcal{X}=\left\{\mathbf{X}^{1}, \ldots, \mathbf{X}^{K}\right\}$ and their associated distributions $\left(f_{s}^{k}, f_{0}^{k}\right)$. In the following experiments, $K=$ 30 .

The first family of tests, $\mathbf{X}^{1}$, calculates the proportion of edge pixels (defined and computed as in [1 by means of an edge oriented integral image) in a window associated with the pose $\Lambda_{i, j}$. That is, $X_{1,1}^{1}$ is the proportion of pixels which are edges within $\Lambda_{1,1}$ and similarly for all $\Lambda_{i, j}$. Test families $\mathbf{X}^{2}$ to $\mathbf{X}^{5}$ are similar to $\mathbf{X}^{1}$, in that they compute the proportion of edge pixels in a window centered on $\Lambda_{i, j}$, but of larger size, by a factor $F=\{2,3,4,5\}$ (see figure1(c)). Note that this factor is different from the scale $S$. Using these pose-indexed tests provides a way to test arbitrarily large regions, even when $\Lambda_{i, j}$ is a small subwindow. These tests also allow for overlapping $\Lambda_{i, j}$ regions and more precise estimation of the face scale.

Families $\mathbf{X}^{6}$ to $\mathbf{X}^{9}$ are similar to $\mathbf{X}^{1}$ but compute the proportion of edge pixels in a particular direction (four possible directions). Similarly to families $\mathbf{X}^{2}$ to $\mathbf{X}^{5}$, families $\mathbf{X}^{10}$ to $\mathbf{X}^{25}$ allow for a scale factor for tests in a particular di- 


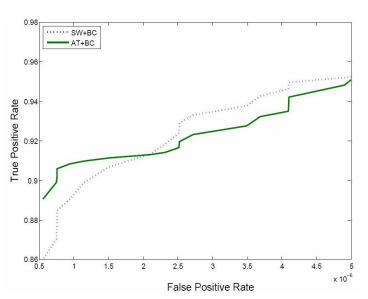

(a)

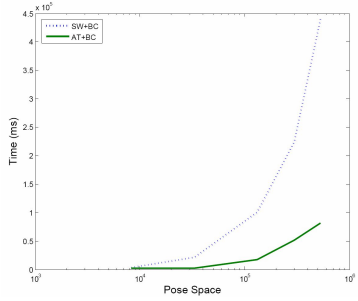

(b)

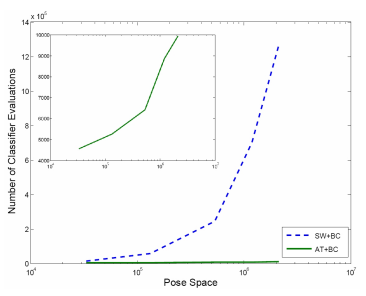

(c)

Figure 3: (a) ROC curve of both $\mathrm{SW}+\mathrm{BC}$ and $\mathrm{AT}+\mathrm{BC}$ to find a face in the Caltech Frontal Face dataset. The performance of both methods is approximately identical. (b) Average computation time with varying pose space size. Note that image size is in logarithmic scale. The AT algorithm performs in almost logarithmic time compared to SW. (c) Average number of classifier evaluations when the pose space increases. Additionally, a zoom of the AT performance is provided.

rection (4 directions $\times 4$ factors). Using integral images allows for computation of these tests with only 4 additions, making them very efficient.

We choose to model all the $f_{s}^{k}$ for $s \in\{0, \ldots, M\}$ using Beta distributions. The Beta family permits to model a wide range of smooth distributions over the interval $[0,1]$ with only two parameters. The parameters of each distribution are determined offline from a small training dataset where the face location and scale is known (more details are given in Section 3.2).

Finally, families $\left\{\mathbf{X}^{26}, \ldots, \mathbf{X}^{30}\right\}$ are the perfect tests and involve testing for a face using a Boosted Cascade (BC). Each family specifies testing for a face at all scales within a given interval $(s \in\{1, \ldots, M\})$. For each interval, we test for face sizes in increments of $10 \%$ of the smallest face size (total of 13 face sizes in the range $[100,300])$. In terms of operations, evaluating this test requires on average 56 additions, 1 multiplication and 1 comparison, per face size, making it significantly more costly than other queries. Since the BC is only informative when the pose is very specific, we restrict this test to leaves in $\Lambda$. These BCs are trained and provided by $\mathrm{OpenCv}$ [12, but modified to restrict testing to specific regions and face sizes. Even though better classifiers have recently been developed, we choose this one as it is publicly available and widely used.

\subsection{Offline Training}

We choose to model each $f_{s}^{k}(\cdot, i)$ with a Beta distribution with parameters $(\alpha, \beta)$. To do this, we randomly selected 50 images, from the Caltech Frontal Face Dataset [26]. Note that far fewer images are used for training here when compared to other search methods (see [15, 28]) which typically use on the order of $10^{3}$ images to train their systems. The estimation of the $f_{s}^{k}(\cdot, i)$ parameters is broken into two parts.

We first estimate all the background densities. That is, for each $k$ and $i$, we randomly select $100 j$ 's per image, such that the face center is not in $\Lambda_{i, j}$. 
We then compute the tests $X_{i, j}^{k}=x$, and use these to compute the parameters using maximum likelihood estimation with 5000 datapoints.

To estimate the foreground densities, a similar procedure is used. We describe the case $s=1$. For each $k$ and $i$, we randomly select $100 j$ 's in each image such that the face center is in $\Lambda_{i, j}$. The parameters of $f_{1}^{k}(\cdot, i)$ are then estimated from the tests $X_{i, j}^{k}=x$. As before 5000 datapoints are used to estimate $(\alpha, \beta)$. In order to estimate $f_{s}^{k}(\cdot, i)$ for $1<s \leq 4$, we subsample the images and repeat the same procedure (similar to [6]). Additionally, the $\int\left(f_{s}^{k}-f_{m}^{k}\right)^{2}$ term from equation (10) is then calculated by using a Monte Carlo approximation, and stored in a look-up table.

\subsection{Single Face Localization}

We setup the AT algorithm with BCs $(\mathrm{AT}+\mathrm{BC})$ to run until a face is found or until $5 \times 10^{5}$ classifier evaluations have been performed (see figure 4 for details on how this was chosen). We compare this with a sliding window approach using the identical $\mathrm{BCs}(\mathrm{SW}+\mathrm{BC})$ and letting it run until a face is found or until all poses have been observed. Note that both $(\mathrm{AT}+\mathrm{BC})$ and $(\mathrm{SW}+\mathrm{BC})$ have the same pose space: all pixels and face sizes $($ e.g. pose space size $=896 \times 592 \times 13=$ $6895616)$. In order to avoid any unfair bias as to where faces may be located, we randomly pick initial starting locations in the image for $(\mathrm{SW}+\mathrm{BC})$, looping around the image in order to observe all the poses. We report that $(\mathrm{AT}+\mathrm{BC})$ allows for exponential computational gains over the sliding window approach while keeping similar performance levels.

Figure 2 shows a typical behavior of the AT algorithm on a given image. In general, the order in which queries are posed is complex and in some cases counter-intuitive - validating the need for an online search strategy.

In figure 3(a) we compare the accuracy of $(\mathrm{AT}+\mathrm{BC})$ and $(\mathrm{SW}+\mathrm{BC})$ on the remaining unused 400 images of the dataset using a $\mathrm{ROC}$ curve. We observe that generally $(\mathrm{AT}+\mathrm{BC})$ does not suffer much from a loss in performance compared to the brute force sliding window approach. Note that the difference between the two methods is not significant.

To compare how much time $(\mathrm{AT}+\mathrm{BC})$ and $(\mathrm{SW}+\mathrm{BC})$ take to locate a face depending on the size of the pose space, we randomly selected a subset of 50 images from the testing set, subsampled these to have images of sizes $(112 \times 74$, $224 \times 148,448 \times 296,672 \times 444,896 \times 592)$. Figure $3(\mathrm{~b})$, shows the average time of both methods for each image size. Note that the overhead of $(\mathrm{AT}+\mathrm{BC})$ - the time to evaluate all queries tested, the update mechanism and the query selection - is included in this plot (the additional time to compute an integral image for oriented edges is not included as it is negligible). As expected, we see that $(\mathrm{SW}+\mathrm{BC})$ is linear in the number of poses. However, the total time $(\mathrm{AT}+\mathrm{BC})$ takes to complete is significantly lower than $(\mathrm{SW}+\mathrm{BC})$ and even more so at large image sizes. In fact, $(\mathrm{AT}+\mathrm{BC})$ remains almost logarithmic even as the number of poses increases. This suggests that AT uses a form of "Divide and Conquer" search strategy. Note, that at image sizes smaller than $(112 \times 74)$, $(\mathrm{AT}+\mathrm{BC})$ is slower than $(\mathrm{SW}+\mathrm{BC})$ due to the overhead. 


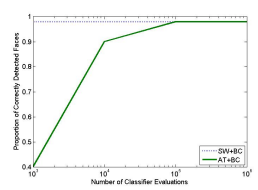

(a)

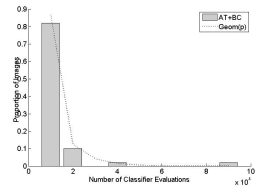

(b)

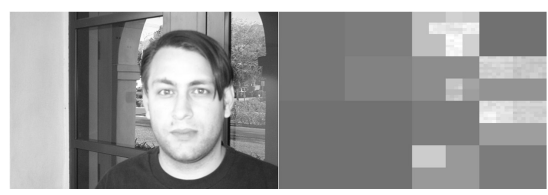

(c)

Figure 4: (a) The proportion of faces detected increases with the number of classifier evaluations: $90 \%$ of faces are correctly detected with only $10^{4}$ evaluations and with $10^{5}$ classifier evaluations, the AT algorithm performs as well as SW, but much faster. (b) Histogram of the number of classifier evaluations. The dotted black line represents the point mass function of the Geometric distribution with parameter $p=1 / 9248$. (c) Face image and associated computation image. This gray scaled image indicates the number of times each pixel has been included in a queried window.

Figure 3(c) shows the average number of classifier evaluations both (AT+BC) and $(\mathrm{SW}+\mathrm{BC})$ perform, when changing the image size. Notice that the difference between $(\mathrm{AT}+\mathrm{BC})$ and $(\mathrm{SW}+\mathrm{BC})$ is even larger than the difference reported in figure 3(b), and that the AT algorithm significantly reduces the number of classifier evaluations. For the largest image size AT requires 100 times fewer evaluations than SW.

In figure 4(a) we show how the accuracy of $(\mathrm{AT}+\mathrm{BC})$ is affected by the total number of classifier evaluations allowed. The dotted line indicates the performance of $(\mathrm{SW}+\mathrm{BC})$ when the entire pose space is observed. We see that after observing the entire pose space $\left(O\left(10^{6}\right)\right.$ evaluations), $98 \%$ accuracy is achieved. Performance results are shown when $(\mathrm{AT}+\mathrm{BC})$ is stopped when either a face has been located or after $\left(10^{3}, 10^{4}, 10^{5}, 10^{6}\right)$ classifier evaluations have be performed. After only $10^{4}$ classifier evaluations nearly $90 \%$ of detectable faces are found. By $10^{5}$ evaluations AT performs at the same accuracy level as SW. In general, we can see in figure 4(b) that the number of evaluations required is approximatively $\operatorname{Geometric}\left(p=10^{-4}\right)$. Hence, on average 0.0014 of the total pose space is evaluated by the classifier.

As in [1, figure 4(c) shows a randomly selected test image, and the corresponding computational image associated (right). The computational image is a gray scale image, which indicates the number of times each pixel has been included in a queried window (all types of queries included). Darker regions show areas where little computation has taken place, while white regions shows important computation. As expected, we can see that regions of the image which contain few features (left part of the image) are not considered for much computation.

\section{Face Detection and Localization}

We now test the AT algorithm in a much harder setting - a detection and localization task. We do this by looking for faces in the MIT+CMU dataset 
23. This dataset contains 130 images, of various sizes, where some images contain no faces, and others contain an unknown number of faces. Face sizes range between 20 pixels to the width of images. As in the previous experiment, we initialize the AT algorithm similarly to that in section 2 and 3 .

To find multiple face instances, we assume that at any point in time, the remaining number of faces to be found in an image follows a Poisson distribution with parameter $\lambda Q$, where $Q$ is the number of pixels unobserved in the image, and $\lambda$ is a face rate. We have chosen $\lambda=10^{-4}$, corresponding to one face per 100x100 pixel image on average and hence $\pi_{0}\left(\bar{\Lambda}_{1,1}\right)=e^{-\lambda Q}$. We then run the AT algorithm until $\pi_{t}\left(\Lambda_{1,1}\right)<\epsilon=10^{-5}$. When a face is found: edges from the detected face region are removed from the integral images and the remaining poses are assigned uniform probability. The algorithm is then restarted with the updated $\pi_{0}\left(\bar{\Lambda}_{1,1}\right)$.

Figure 5(a) shows the ROC curve of both the $(\mathrm{AT}+\mathrm{BC})$ and $(\mathrm{SW}+\mathrm{BC})$ methods on the MIT $+\mathrm{CMU}$ dataset. In both cases no post-processing step was applied to these results (i.e. No Non-Maximum suppression). First we note that the MIT+CMU testset is much harder than the Caltech Frontal Face set. In general, the performance of the AT algorithm is comparable to the brute force approach. There is, however, a slight performance decrease in $(\mathrm{AT}+\mathrm{BC})$ when compared to the exhaustive search. That is, we notice that even though the classifier used (BC) is not very good (when compared to state-of- the-art classifiers), little accuracy loss is observed when used in the AT framework.

From this experiment, $(\mathrm{AT}+\mathrm{BC})$ required $O\left(10^{8}\right)$ classifier evaluations over the entire testset, while $(\mathrm{SW}+\mathrm{BC})$ required $O\left(10^{9}\right)$ evaluations. Figure $5(\mathrm{~b})$, shows the number of classifier evaluations required by both $(\mathrm{AT}+\mathrm{BC})$ and $(\mathrm{SW}+\mathrm{BC})$ on each image. Generally, we see that AT is still able to significantly reduce the total number of evaluations required even though the number of faces in the images is apriori unknown. Figure 5(c), shows a similar result in terms of time. Again, computational gains are of one order of magnitude over the entire testset.

Notice in figures $5(\mathrm{~b})$ and 5 (c) that for images of the same pose space size, the number of classifier evaluations and time necessary for $(\mathrm{AT}+\mathrm{BC})$ to terminate vary. This variance is due to the fact that $(\mathrm{AT}+\mathrm{BC})$ stops when the estimate of having a face in the image is very low: $\pi_{t}\left(\Lambda_{1,1}\right)<\epsilon=10^{-5}$. Hence, in images which contain many face-like features, the algorithm will need to visit many more locations to see if faces are still present. This is precisely what is observed in figures $5(\mathrm{~b})$ and $5(\mathrm{c})$.

\section{Conclusion}

We have proposed an Active Testing framework in which one can perform fast face detection and localization in images. In order to find faces, we use a coarseto-fine method, while sampling subwindows which maximize information gain. This allows us to quickly find the face pose by focusing on regions of interest, and pruning large image regions. We show through a series of experiments, that 


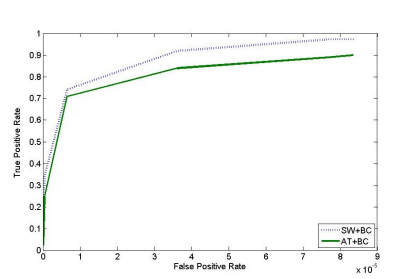

(a)

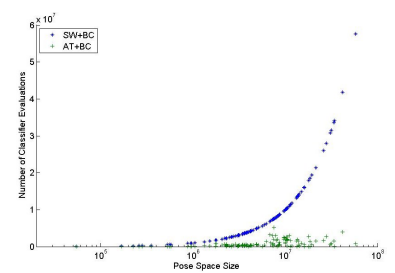

(b)

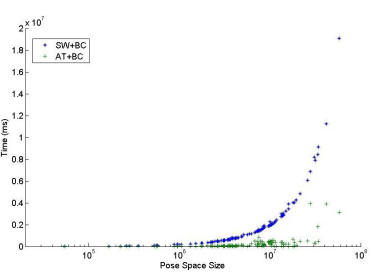

(c)

Figure 5: (a) ROC for both the sliding window and the Active Testing approaches on the MIT+CMU frontal face dataset. The AT algorithm achieves similar performance levels to the exhaustive search. (b) Number of classifier evaluations for each image in the testset. Clearly the AT approach does not suffer as much from the increase in pose space. (c) Time performance for each image in the testset.

the active testing framework can be used to significantly reduce the number of classifier evaluations when searching for an object. Exponential speedup is observed when detecting and locating faces compared to the traditional sliding window approach (particularly on large image sizes), without significant loss in performance levels, indicating that this method is scalable to larger image sizes.

\section{Acknowledgments}

Funding for this research was provided in part by NIH Grant 1 R01 EB 00796901 and the Duncan Fund for the Advancement of Statistics Research, Award 08-19.

\section{References}

[1] Y. Amit and D. Geman. A computational model for visual selection. Neural Computation, 11:1691-1715, 1999.

[2] V. Belle, T. Deselaers, and S. Schiffer. Randomized trees for real-time onestep face detection and recognition. International Conference on Pattern Recognition, 2008.

[3] N. Dalal and B. Triggs. Histograms of oriented gradients for human detection. IEEE Conference on Computer Vision and Pattern Recognition, pages 886-893, 2005.

[4] L. Ding and A. M. Martinez. Features versus context: An approach for precise and detailed detection and delineation of faces and facial features. IEEE Transactions on Pattern Analysis and Machine Intelligence, 99(PrePrints), 2010. 
[5] M. Everingham, L. Van Gool, C. K. I. Williams, J. Winn, and A. Zisserman. The PASCAL Visual Object Classes Challenge 2009 (VOC2009) Results. http://www.pascalnetwork.org/challenges/VOC/voc2009/workshop/index.html.

[6] F. Fleuret and D. Geman. Coarse-to-fine face detection. International Journal of Computer Vision, 41:85-107, 2001.

[7] F. Fleuret and D. Geman. Stationary features and cat detection. Journal of Machine Learning Research, 1, 2008.

[8] D. Geman and B. Jedynak. Shape recognition and twenty questions. INRIA Technical Report 2155, 1993.

[9] D. Geman and B. Jedynak. An active testing model for tracking roads from satellite images. IEEE Transactions on Pattern Analysis and Machine Intelligence, 18(1):1-14, January 1996.

[10] C. Gracia and M. Delakis. Convolutional face finder: A neural architechture for fast and robust face detection. IEEE Transactions on Pattern Analysis and Machine Intelligence, 26(11):1408-1423, 2004.

[11] T. Hastie, R. Tibshirani, and J.H. Friedman. The elements of statistical learning. August 2001.

[12] Intel. Opencv open source computer vision library.

[13] X. Jiang, B. Mandal, and A. Kot. Eigenfeature regularization and extraction in face recognition. IEEE Transactions on Pattern Analysis and Machine Intelligence, 30(3), 2008.

[14] F. De la Torre, J. Campoy, Z. Ambadar, and J.F. Cohn. Temporal segmentation of facial behavior. International Conference on Computer Vision, 2007.

[15] C.H. Lampert, M.B. Blaschko, and T. Hofmann. Beyond sliding windows: Object localization by efficient subwindow search. IEEE Conference on Computer Vision and Pattern Recognition, pages 1-8, 2008.

[16] P. Li and S. Prince. Joint and implicit registration for face recognition. IEEE Conference on Computer Vision and Pattern Recognition, 2009.

[17] S.Z. Li and Z. Zhang. Floatboost learning and statistical face detection. IEEE Transactions on Pattern Analysis and Machine Intelligence, 26(9), 2004.

[18] D. Lowe. Distinctive image features from scale-invariant keypoints. International Journal of Computer Vision, 60(2):91-110, 2004. 
[19] M. Osadchy, Y. LeCun, and M. Miller. Synergistic face detection and pose estimation with energy-based models. Journal of Machine Learning Research, 8:1197-1215, 2007.

[20] E. Osuna, R. Freund, and F. Girosi. Training support vector machines: an application to face detection. IEEE Conference on Computer Vision and Pattern Recognition, pages 130-136, 1997.

[21] H.A. Rowley, S. Beluja, and T. Kanade. Human face detection in visual scenes. Advances in Neural Information Processing Systems, pages 875881, 1996.

[22] H. Schneiderman. Feature-centric evaluation for efficient cascaded object detection. IEEE Conference on Computer Vision and Pattern Recognition, 2004 .

[23] H. Schneiderman and T. Kanade. Frontal face images. 2000.

[24] S. Shah, S. H. Srinivasan, and S. Sanyal. Fast object detection using local feature-based svms. In $M D M$, pages 1-5, 2007.

[25] P. Viola and M. Jones. Robust real-time face detection. International Journal of Computer Vision, 57(2):137-154, 2004.

[26] M. Weber. Frontal face dataset, california institute of technology. http://www.vision.caltech.edu/html-files/archive.html, 1999.

[27] S.Y. Yan, S.G. Shan, X.L. Chen, and W. Gao. Locally assembled binary (lab) feature with feature-centric cascade for fast and accurate face detection. IEEE Conference on Computer Vision and Pattern Recognition, pages $1-7,2008$.

[28] J. Yuan, Z. Liu, and Y. Wu. Discriminative 3d subvolume search for efficient action detection. IEEE Conference on Computer Vision and Pattern Recognition, 2009. 